\title{
Instabilities, coherency, and spectra of the NovoFEL radiation
}

\author{
V.V. Kubarev ${ }^{1,2}$ \\ ${ }^{1}$ Budker Institute of Nuclear Physics, Novosibirsk, Russia, V.V.Kubarev@inp.nsk.su \\ ${ }^{2}$ Novosibirsk State University, Novosibirsk, Russia
}

\section{Introduction}

Novosibirsk free electron laser is user facility consists of three lasers of terahertz $(240-90 \mu \mathrm{m})$, far infrared $(80-30 \mu \mathrm{m})$, and infrared $(30-5 \mu \mathrm{m})$ ranges [1]. The NovoFEL spectrum is one of the main parameter for many user applications. Spatially it is important for ultra-fast high-resolution spectroscopy and radar applications developing on the NovoFEL. The NovoFEL spectra depend on both temporary form of its radiation (continuous trains of 30-150 ps pulses with repetition frequencies of 5.6 $\mathrm{MHz}$ ) and different radiation instabilities in the device. In the case of full intra-pulse coherency we have narrowest line of width equal to inverse pulse duration (Fourier limit). Coherency between close pulses causes splitting of the spectrum on many spectral components with frequency difference of $c / 2 L(5.6 \mathrm{MHz})$, where $L$ is optical resonator length. Spectral width of the components is inverse proportional to total coherency length between pulses. On the one hand the coherency must be not less than $L \times Q$-factor of optical resonator due to laser nature of FEL radiation. Moreover in the case of ideal laser coherency length can be much more than the value. But some accumulative phase shift between fields in pulses can take place in conditions of real experiment due to different instabilities. Thus obtaining of new experimental data about fine structure of the NovoFEL radiation is the main subject of this paper.

Other large-scale spectral peculiarities $(\sim \mathrm{GHz})$ of the NovoFEL radiation caused by different fast instabilities were investigated early in works [2-7]. We found two types of side-band modes caused by classical FEL instability on trapped electron $[3,5,7]$ and by some instability of an unknown nature when the NovoFEL pulses have two-humped form $[2,6,7]$. These mode structures were measured both in a relatively stable regime $[2,3,5,7]$ and a strongly unstable regime $[6,7]$, where we used our original method of one-pulse spectral measurements. Full stabilization of all side-band instabilities was also demonstrated by using stabilization frequency detuning between electron and light pulses [3,5-7].

Though the fine spectral structure has to be in all the NovoFEL regimes, its parameters can be strongly different in stable and unstable cases. That is other main task of the investigation.

\section{Experimental setup}

Two experimental setups we plane to use in the study. The first one is ultra-long resonance waveguide vacuum Fabry-Perot interferometer (FPI) with calculated resolution $(1.5-7) \cdot 10^{6}$ (Fig. 1). It should be noted that its resolution is higher than resolution of the other our spectral devices (Fourier spectrometer, grating spectrometer) by factor of $10^{3}-10^{4}$.
If we will find a fine structure with spectral width smaller than $0.5 \mathrm{MHz}$, than we will use a second setup with spectral resolution of few $\mathrm{kHz}$, based on mixing of the NovoFEL radiation with radiation of ultramonochromatic terahertz gas laser $[8,9]$ in high-sensitive fast Schottky diode detector [10].

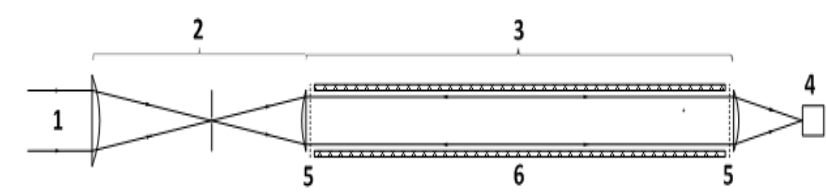

Fig. 1. Optical scheme of installation for measuring of fine spectral structure of the NovoFEL radiation by ultra-long waveguide vacuum Fabry-Perot interferometer: 1 - NovoFEL beam, 2 - beam transformer, 3 - Fabry-Perot interferometer, 4 lock-in detector, 5 - electroformed mesh, 6 - hollow dielectric waveguide. Length of Fabry-Perot interferometer is $2659 \mathrm{~mm}$ (1/10 part of the NovoFEL optical resonator length)

\section{Experimental results}

Now we obtained only first results with start version of our FPI with nickel meshes. Its calculated spectral resolution was $1.5 \cdot 10^{6}$.

Fig. 1 illustrates importance of exact tuning of resonance length of our FPI. This tuning was made by special mechanical system moving one of the mesh mirrors. Fine displacement was carried out by heating of hollow invar rods of the FPI by circulating thermo-stabilized liquid.

Fine structure of the NovoFEL radiation is shown in Fig. 3 and Fig. 4 for resonance (a) and stabilized (b) regimes.

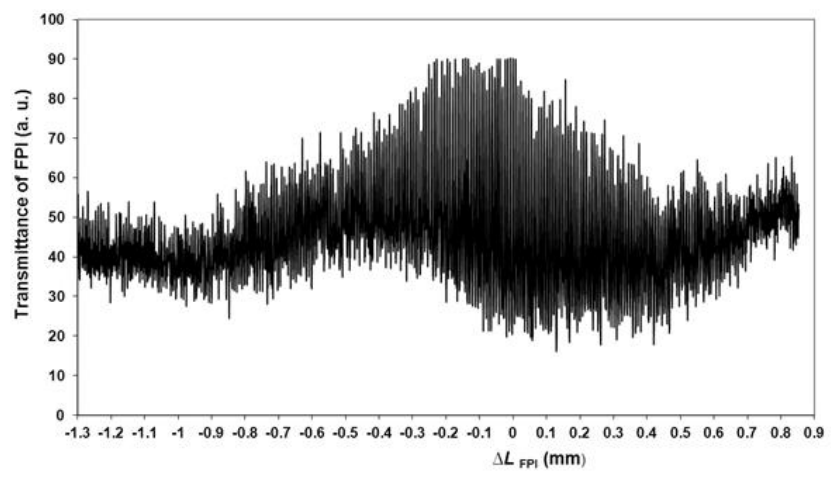

Fig. 2. Transmittance of FPI versus difference of its length to resonance length equal to $1 / 10$ part of NovoFEL optical resonator. Radiation wavelength $-168 \mu \mathrm{m}$

For stabilized regimes (b) spectral resolution was practically equal to calculated apparatuses function of our FPI with nickel meshes. Thus the resolution should be increased for correct measurement of real fine structure in the regimes. 

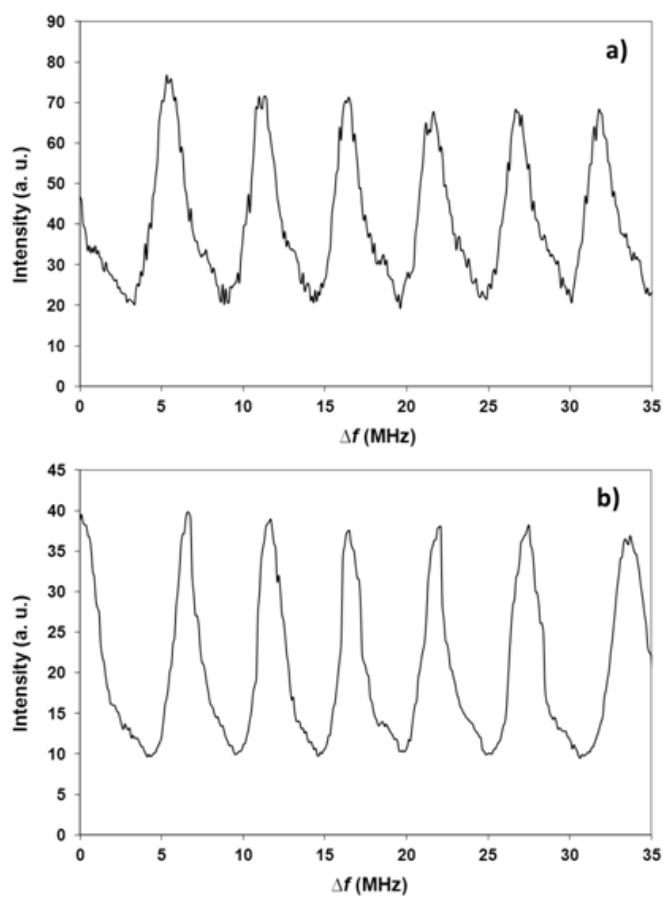

Fig. 3. Fine structure of the NovoFEL radiation at wavelegth $130 \mu \mathrm{m}(2.3 \mathrm{THz})$ for resonance (a) and stabilized (b) regimes
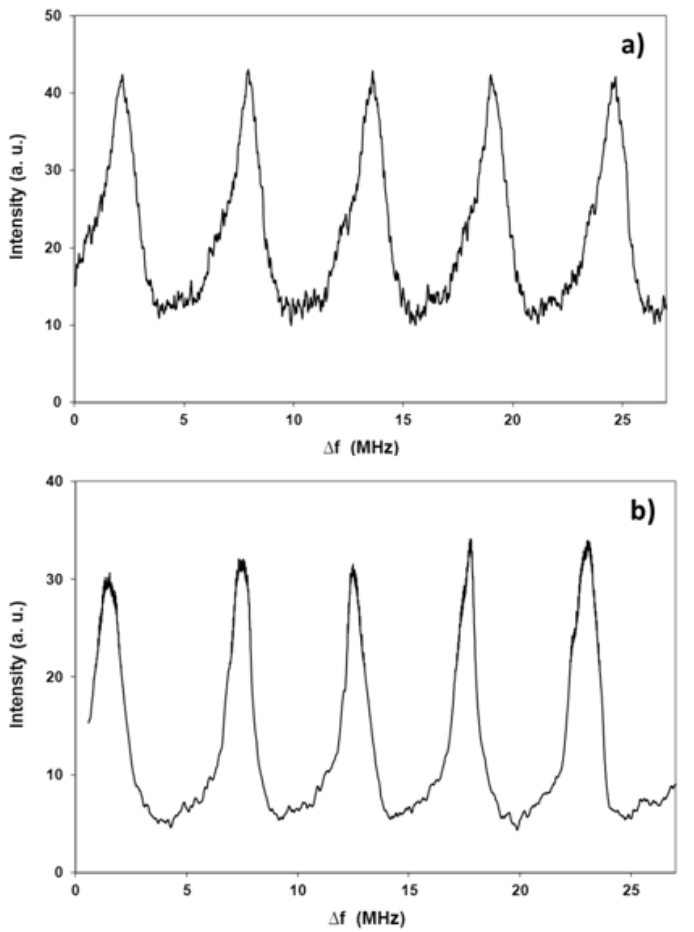

Fig. 4. Fine structure of the NovoFEL radiation at wavelegth $168 \mu \mathrm{m}(1.8 \mathrm{THz})$ for resonance (a) and stabilized (b) regimes
For resonance regimes (a) we can see some broadening of the mode spectral lines. Therefore real fine structure of NovoFEL radiation is order of apparatus function. We can see also that the broadening look like appearing of weak transverse modes.

We plan to increase spectral resolution of the FPI by 4-5 times making use gold meshes in place of nickel meshes.

\section{References}

1. Kulipanov G.N., Bagryanskaya E.G., Chesnokov E.N., Choporova Yu.Yu., Gerasimov V.V., Getmanov Ya.V., Kiselev S.L., Knyazev B.A., Kubarev V.V., Peltek S.E., Popik V.M., Salikova T.V., Scheglov M.A., Seredniakov S.S., Shevchenko O. A., Skrinsky A. N., Veber S. L., and Vinokurov N. A. Novosibirsk free electron laser - facility description and recent experiments // IEEE Transaction on Terahertz Science and Technology. 2015. V. 5, No.5. P. 798-809.

2. Kubarev V.V., Vinokurov N.A., Kotenkov V.V. et al. Observation of sideband instability in the Novosibirsk terahertz free electron laser // Proc. 31st Int. Conf. IRMMW-THz-2006, Shanhai, China, September 18-22, 2006, P. 415.

3. Kubarev V.V., Kulipanov G.N., Kolobanov E.I., et al. Modulation instability, three mode regimes and harmonic generation at the Novosibirsk terahertz free electron laser // Nuclear Instruments \& Methods in Nuclear Research, 2009. V. A603. P. 25-27.

4. Kubarev V.V., Ovchar V.K., and Palagin K.S. Ultra-fast Terahertz Schottky Diode Detector // Proc. 34th Int. Conf. IRMMW-THz-2009, Busan, Korea, September 21-25, 2009, report 09030439.

5. Kubarev V.V. Detailed observation of modulation instability on THz NovoFEL // Proc. 37th Int. Conf. IRMMW-THz2012, Wollongong, Australia, September 23 - 28, 2012, report Fri-A-4-2.

6. Kubarev V.V. Ultrafast high-resolution spectroscopy of separate NovoFEL pulses // Proc. 38th Int. Conf. IRMMWTHz-2013, Mainz, Germany, September 1-6, 2013, report Mo14-4.

7. Kubarev V.V. Optical system, diagnostics, and experiments on terahertz and infrared free electron lasers // Doctoral Thesis, Novosibirsk, Russia, 2016, http://www.inp.nsk.su/news/ defences.ru.shtml

8. Kubarev V.V. and Kurenskii E.A. Ultralow-noise highpower DCN laser with rf pumping // Quantum Electronics 1996. V. 26, No 4. P. 303-306.

9. Kubarev V.V. and Kurenskii E.A. Very-low-noise $\mathrm{H} 2 \mathrm{O}$ laser with rf pumping // Quantum Electronics 1995. V. 25, No.12. P. 1141-1145.

10. Kubarev V.V., Kazakevich G.M., Jeong Y.U., Lee B.J. Quasi-optical highly sensitive Schottky-barrier detector for a wide-band FIR FEL // Nuclear Instruments \& Methods for Nuclear Research 2003. V. A 507. P. 523-526. 